\title{
Salud mental en desastres naturales
}

\author{
Mental health in natural disasters
}

Celso Bambarén Alatrista ${ }^{1, a, b}$

\section{RESUMEN}

Los desastres se pueden considerar como un problema de salud pública que pueden afectar el comportamiento psicosocial de la población, especialmente de los grupos más vulnerables, como son los niños, adolescentes, mujeres, ancianos y personas con enfermedades físicas y mentales. Las personas desarrollan diferentes tipos de respuestas psicológicas al desastre según su capacidad de superación de la crisis inicial. El personal de salud tiene que identificar y cuidar a las personas que presenten cambios psicológicos que revelen que no se está superando el impacto inicial, y también apoyar la continuidad del tratamiento de las personas con problemas mentales previos al desastre.

PALABRAS CLAVE: Desastres, salud mental, intervenciones psicológicas.

\section{SUMMARY}

Disasters can be considered as a public health problem that could affect the psychosocial behavior of population, especially the most vulnerable groups such as children, adolescents, women, old people and people with physical and mental illness. People develop different types of psychological responses to disasters by their ability to overcome the initial crisis. Health personnel have to identify and look after people with psychological changes which reveal that they are not overcoming the initial impact; and also support the continuity of the people treatment with mental health problems pre-disaster.

KEYWORDS: Disasters, mental health, psychological interventions.

\section{INTRODUCCIÓN}

Los desastres, entendidos como situaciones generadas por sucesos adversos de origen natural o producidos por el hombre, que exceden la capacidad de respuesta de una comuna, región o país, ocasionan efectos en la salud pública debido a que:

- Causan un número inesperado de muertes, lesiones, discapacidad y enfermedades en la zona afectada, que generan una demanda extraordinaria de atención en la red asistencial.

- Afectan y destruyen la infraestructura de los servicios de agua y saneamiento, y de la red de establecimientos de salud.

- Incrementan el riesgo potencial de enfermedades trasmisibles asociadas al deterioro de las condiciones de vivienda y saneamiento, y a la aparición de peligros ambientales.
- Generan grandes desplazamientos de la población.

- Producen cambios en el comportamiento psicológico y social, que pueden generar crisis en las personas afectadas (Noji, 2000).

\section{EFECTOS DE LOS DESASTRES EN LA SALUD MENTAL}

Los desastres afectan la salud mental de una gran parte de la población, ya sea en forma directa, debido a la pérdida de un familiar o a la destrucción de su vivienda; o en forma indirecta, por los efectos inmediatos del suceso que afectan a las personas, pero sin que sufran pérdidas de familiares o de bienes materiales.

Las alteraciones de la salud mental se pueden presentar desde síntomas psicosomáticos, como falta 
o exceso de apetito, problemas digestivos, erupciones cutáneas, náuseas y palpitaciones, y otros síntomas, como dificultad para dormir, estrés, irritabilidad, dificultad para concentrarse, problemas familiares y laborales, y consumo de alcohol y drogas. En la población afectada y en el personal de salud se han observado cambios en el sueño, el apetito, ansiedad, depresión, síndrome de "burn out" o de agotamiento; y en general, manifestaciones del síndrome de stress postraumático. Los síntomas pueden ser variados, dependiendo de la vulnerabilidad de las personas: su experiencia previa al hecho, el soporte social y familiar y el grado y tiempo de afectación. Por lo general, los sucesos inesperados (terremotos) y los conflictos armados generan una situación de estrés prolongado; sin embargo, los de afectación colectiva producen mayor impacto en la población (OPS, 2010)

Los factores que influyen en el impacto psicosocial de los desastres son:

- La naturaleza del fenómeno adverso que produce el desastre, pudiendo diferenciarse entre desastres de inicio súbito, como terremotos y tsunamis; y otros de inicio lento y curso insidioso, como las inundaciones.

- Las características de personalidad de los afectados. Los desastres no afectan a todas personas por igual: algunas tienen mayor probabilidad de desarrollar problemas psicológicos que otras que pueden tener mayor capacidad para enfrentar mejor la situación.

- El entorno y las circunstancias: condiciones ambientales y familiares de las víctimas, y las redes sociales existentes, previas al desastre (Organización Panamericana de la Salud (OPS), 2006).

La afectación post desastre de las personas depende de factores de resiliencia y de riesgo (psicopatología). Varios factores biológicos, sociales, psicológicos anteriores al desastre influyen sobre las personas, determinando si tienen menor o mayor capacidad para resistir a las condiciones nuevas y extremas que pueden generar una situación de emergencia.

Los grupos de personas que están más expuestas son:

- Mujeres embarazadas, madres solteras y viudas.

- Niños y adolescentes, siendo los más vulnerables aquellos que han perdido a su familia.

- Personas desempleadas.

- Ancianos, especialmente si han perdido a las personas que los cuidaban.

- Personas desplazadas de su lugar de vivienda.

- Personas que pierden su sistema de apoyo social y psicológico.
- Personas que sufrían enfermedades físicas o mentales en el momento del desastre (IASC, 2007)

\section{Erupciones volcánicas}

El volcán, a diferencia de otros desastres, es una amenaza física y visible. La posibilidad de una erupción volcánica genera tensión, ansiedad, depresión, pesadillas, neurosis y otras manifestaciones de estrés (Zeballos, Meli, Vilchis y Barrios, 1996). La actividad volcánica puede durar meses o años, lo que origina una actitud aprehensiva de largo tiempo dominada por la tensión y el temor en la población en el área de riesgo del volcán. Por otra parte, la contaminación ambiental, generada por la actividad del volcán, puede favorecer la aparición de manifestaciones psíquicas como consecuencia directa de la afectación del sistema nervioso central, o como respuesta emocional (OPS, 2005).

\section{Inundaciones}

Los estudios realizados en las personas y comunidades afectadas por inundaciones muestran que las alteraciones psicológicas severas no son frecuentes; lo más común son los problemas emocionales leves y transitorios. Los resultados de los estudios revelaron que las inundaciones precipitaron las reacciones psicológicas al estrés y al esfuerzo. Los grupos vulnerables, como los adultos mayores y las personas muy jóvenes, están en mayor riesgo de presentar reacciones psicológicas. Algunos estudios han reportado el incremento de los casos de ansiedad, depresión e insomnio entre las víctimas de las inundaciones (Ahern, Kovats, Wilkinson, Few y Matthies, 2005). También se ha encontrado el incremento del riesgo de gastroenterocolitis asociado a la profundidad de la inundación, y al incremento de stress psicológico en adultos (Reacher, McKenzie, Lane, Nichols y Kedge, 2004). Por otra parte, también se ha observado cambios de conducta en niños, como también un incremento de casos de comportamientos agresivos y enuresis (Durkin, Khan, Davidson, Zaman y Stein, 1993).

\section{Terremotos}

Este fenómeno natural, es probablemente, el que genera los mayores efectos en la salud mental de la población, y en la infraestructura social y productiva de la zona donde se produce el sismo. A continuación se resumen los efectos en la salud mental de la población en algunos de los más desastrosos terremotos ocurridos en América. 


\section{Terremotos en El Salvador}

El 13 de enero de 2001, El Salvador fue afectado por un sismo de magnitud 7,9 en la escala de Ritcher, que generó cuantiosos daños personales y materiales. Se observó que el $20 \%$ de la población afectada indirectamente requirió de algún tipo de apoyo psicológico, mientras que los afectados en forma directa, necesitaban de una atención prolongada, que no podía ser atendida por el sistema sanitario de El Salvador debido a su limitada capacidad de respuesta para atender los problemas de salud mental.

Entre las reacciones emocionales más frecuentes que se encontraron fueron: tristeza, depresión, intentos de suicidio, ansiedad, síndrome de estrés postraumático, desorganización personal y conductas violentas. Gran parte de los afectados sufrieron la desorganización de sus núcleos familiares por pérdida total, o parcial de los miembros de la familia. Quedó un número considerable de huérfanos (menores de edad), de personas viudas y de personas de la tercera edad abandonadas (OPS, 2002).

La situación psicosocial de la población afectada se agravó debido al sismo del 13 de febrero de magnitud 6,6 Ritcher y a los dos sismos de menor magnitud del 17 de febrero y del 28 de marzo, acontecimientos que reavivaron las reacciones de angustia y de pánico, y los casos de ansiedad, depresión, insomnio, erupciones cutáneas, diarrea y dolores de estómago causados por el estrés. Se registraron más de 8000 consultas por depresión y trastornos de ansiedad, problemas que afectaron también al personal de salud (Comisión Económica para América Latina y el Caribe (CEALC), 2001). Además, el sismo de febrero y las continuas réplicas posteriores, generaron que la población perdiese la confianza en las explicaciones de los científicos acerca de la persistencia de las mismas (OPS, 2002).

\section{Terremoto en Pisco - Perú}

El 15 de agosto de 2007, un terremoto de magnitud 7,9 Mw afectó la costa central de Perú y produjo daños humanos y materiales en las regiones de Ica y de Lima y afectó también a la Región Huancavelica. Según la información oficial, el terremoto produjo 596 muertos, más de mil heridos y 464,314 damnificados. En la ciudad de Chincha, una de la más afectada por el terremoto, entre los meses de agosto y diciembre de 2007, se registraron 423 atenciones: $48 \%$ fueron por ansiedad; el $23 \%$, por trastorno depresivo; y el
$25 \%$, por cefalea tensional. Posteriormente, en el trimestre de 2008, se registraron 214 atenciones: el $51 \%$ fue por ansiedad; el $18 \%$, trastorno depresivo; el $9 \%$, por abuso de alcohol-drogas; y el 3\%, de intentos de suicidio. Los problemas de salud mental se presentaron en mujeres entre los veinte a cuarenta y nueve años de edad. En la ciudad de Pisco, el mayor número de atenciones de salud mental fue por ansiedad, trastornos depresivos y violencia familiar. En los meses posteriores, el número de atenciones de salud mental en los hospitales de Chincha y Pisco disminuyó, se mantuvieron los problemas de ansiedad y depresión como las principales causas de atención (OPS, 2010).

\section{Terremoto en Haití}

El 12 de enero de 2010, un sismo de magnitud 7,0 Mw devastó Haití, produjo la muerte de 220000 personas, 300000 heridos y dejó sin vivienda a 1,5 millones de personas. Este terremoto agravó las vulnerabilidades y problemas de salud mental preexistentes. Se registró un incremento del $2 \%$ al $3 \%$ de los casos de trastorno mental grave (psicosis, estrés post traumático severo y otras patologías) en relación a la situación previa al sismo; mientras que los trastornos leves y moderados, incluyendo la ansiedad, el estrés post traumático moderado - leve y los cambios del estado de ánimo aumentaron en un $10 \%$ en relación a la situación antes del desastre (Pan American Health Organization (PAHO), 2011).

\section{Terremoto en Chile}

El 27 de febrero de 2010, un terremoto de magnitud de 8,8 en la escala de $\mathrm{Mw}$ afectó gran parte del territorio chileno, donde viven más de trece millones de personas. El sismo fue seguido de un tsunami que afectó a numerosas comunas ubicadas en el borde costero. Se estima que 512 personas fallecieron, 500 mil viviendas quedaron con daño severo y hubo dos millones de personas damnificadas. Este terremoto ocasionó daños severos en la red asistencial, principalmente hospitalaria, donde se perdieron el $22 \%$ de las camas psiquiátricas disponibles en el país, y dos hospitales psiquiátricos (El Peral y Pinel) quedaron con daños graves.

La revisión de las estadísticas de atenciones y su comparación con los datos de los tres años previos, permitió observar que el número de atenciones del trastorno de ansiedad se incrementó levemente en los meses post terremoto en las regiones afectadas por el 
desastre. Sin embargo, se debe tomar en cuenta que los casos de somatizaciones, asociada al fenómeno natural, pueden fácilmente quedar registrados solo como molestias orgánicas sin ninguna vinculación con los efectos del desastre (OPS, 2010).

\section{RESPUESTAS PSICOLÓGICAS A LOS DESASTRES}

Las personas afectadas por los desastres tienen diferentes tipos de respuestas psicológicas: algunas se hacen evidentes en las primeras setenta y dos horas después del desastre, como crisis emocionales, incremento de la excitación, apatía, ansiedad difusa y temor a conocer la realidad. Al primer mes, se observa duelo, tristeza, aflicción, miedo, manifestaciones somáticas diversas, irritabilidad, pérdida de sueño y apetito, y problemas en el desempeño laboral y las relaciones interpersonales. Posteriormente, a los dos o tres meses, se evidencia tristeza, manifestaciones somáticas, ansiedad, y pérdida de confianza debido a dificultades en la adaptación o ante la falta de solución a sus problemas derivados del desastre. A partir de los tres meses, se podría observar duelos patológicos, conductas que indican riesgo de suicidio, síntomas de estrés postraumático, fatiga crónica, inhabilidad para trabajar y apatía.

En los casos de situación traumática generada por el desastre y desborde la capacidad de la persona, se puede presentar una crisis psicológica, denominada desorden de estrés agudo, que se caracteriza por miedo intenso, impotencia, horror, ausencia total de emociones, sentimiento de desconexión, sentido de irrealidad y amnesia. Aquellas personas que sufren de este tipo de desorden, se muestran ansiosas, excitables, agitadas, irritables y desesperanzadas; además, presentan sueños recurrentes con escenas repetitivas y recuerdos del hecho traumático, dificultad para concentrarse, sentimientos de culpa por haber sobrevivido, y dificultades para integrarse a su medio laboral y familiar. Cuando la persona es diagnosticada y tratada oportunamente, los síntomas disminuyen o desaparecen al mes. En los otros casos, los síntomas persistirán, y si la duración está entre uno a tres meses, se le denomina desorden de estrés post traumático, pudiéndose convertir en crónico cuando las manifestaciones están presentes por más de tres meses (OPS, 2002).

\section{Intervenciones psicológicas post desastre}

Son los procesos que sirven para ayudar a una persona o familia a sobrellevar un hecho traumático, de modo que haya menos consecuencias poco saludables o desorganizantes, y se manifieste una mayor probabilidad de crecimiento y superación. Las cuatro fases de las intervenciones son:

- Fase pre-crítica: corresponde a las horas o días previos al desastre, lo cual es válido para aquellas situaciones que pueden ser anticipadas. Las intervenciones se orientan a las medidas de protección preventiva, a fin de prevenir o mitigar los daños producidos por el potencial desastre.

- Fase crítica: durante el desastre y las setenta y dos horas posteriores. Las intervenciones se focalizan en protección de la seguridad física, satisfacción de necesidades básicas, control de la desorganización social, orientación sobre las reacciones psicosociales, evaluación rápida de necesidades emocionales y primera ayuda psicológica por personal no especializado.

- Fase post-crítica: puede durar de uno a seis meses, dependiendo del tipo de desastre y de la población afectada. Las intervenciones se focalizan en apoyar a la organización de la propia comunidad, coordinación interinstitucional, educar y capacitar sobre salud mental, atención psicosocial para grupos vulnerables, intervención en crisis y atención de las personas con trastornos mentales.

- Fase de recuperación: seis meses posterior a la tragedia que puede prolongarse hasta por cinco años en emergencias complejas. Las intervenciones más importantes son el desarrollo de proyectos ocupacionales y productivos, la continuidad de organización comunitaria y la coordinación interinstitucional, así como de la educación y capacitación, y atención de las personas con trastornos mentales.

Un gran número de intervenciones puede ser desarrollado por personal no especializado que está entrenado para identificar y atender las más frecuentes manifestaciones psicológicas que se presentan después de un desastre. Las intervenciones a cargo de un especialista son, básicamente, aquellas orientadas a personas que presentan trastornos de conciencia persistente, psicosis, riesgo suicida alto, complicaciones graves por abuso de alcohol o drogas, y problemas que no han podido ser solucionados por el personal no especializado.

Las principales intervenciones que debe desarrollar el personal no especializado son:

- Intervenciones comunitarias preventivas, que incluyen información y orientación, actividades 
grupales con niños y adolescentes, grupos de apoyo emocional y desarrollo de grupos de ayuda mutua.

- Intervenciones preventivas con personas que presentan reacciones emocionales, duelos $\mathrm{o}$ conductas violentas de mayor intensidad, aplicando métodos individuales y grupales.

- Intervenciones individuales y grupales con personas que presentan trastornos, como ansiedad, crisis de pánico, depresión, trastornos de somatización, trastorno de estrés postraumático, y abuso de alcohol o drogas (Benveniste, 2000; Asociación de Capacitación e Investigación para la Salud Mental (ACIPLSM), 2001).

En situaciones de desastres, es importante que el personal de salud y los integrantes de la comunidad aprendan a identificar oportunamente a las personas que presenten algún problema psicosocial, observando especialmente a los grupos más vulnerables. También, es importante contar con información previa de las personas que tienen problemas mentales (OPS, 2010).

En el caso del terremoto de Pisco, se realizaron talleres psico-educativos, se formaron grupos operativos para ayuda mutua y se establecieron programas de capacitación para agentes de salud, docentes y líderes de organizaciones sociales para abordar el caso de salud mental en situaciones de desastre. Varias organizaciones realizaron acciones para prevenir el desarrollo del síndrome de stress postraumático y conductas sociales negativas, (abuso de sustancias tóxicas y violencia familiar y social) abuso de sustancias tóxicas y violencia familiar y social.

En la etapa crítica post terremoto de Chile, los equipos de atención de salud mental realizaron acciones de protección de la salud mental en albergues y otras comunidades con alta vulnerabilidad, y se prestaron atenciones de primera respuesta a personas con desbordes emocionales o trastornos mentales provocados o agravados por el terremoto (tanto para población general como para funcionarios del sistema público de salud). Se apoyó a personas que se encontraban en tratamiento ambulatorio en el nivel secundario al momento del terremoto, asegurando acceso a psicofármacos, y efectuando visitas domiciliarias a las personas con trastornos mentales más severos. Por otra parte, se reubicaron en sitios seguros a los pacientes que estaban hospitalizados en instituciones con daños estructurales provocados por el terremoto, y se entregó información a la población sobre las reacciones emocionales a desastres y cómo enfrentarlas, a través de folletos y medios de comunicación, inicialmente radioemisoras y pequeñas radios comunitarias, y posteriormente, por la televisión (OPS, 2010).

\section{REFERENCIAS BIBLIOGRÁFICAS}

1- Ahern, M., Kovats, R., Wilkinson, P., Few, R. \& Matthies, F. (2005). Global health impacts of floods: Epidemiological evidence. Epidemiol Rev,27,3646.

2. Asociación de Capacitación e Investigación para la Salud Mental. (2001). Manual de intervención en crisis en situaciones de desastres. El Salvador: Asociación de Capacitación e Investigación para la Salud Mental.

3. Benveniste, D. (2000). Intervención en crisis después de grandes desastres. Revista de la Sociedad Psicoanalítica de Caracas, 8 (1), 20-23.

4. Comisión Económica para América Latina y el Caribe. (2001). El Salvador: Evaluación del terremoto del martes 13 de febrero de 2001 (addendum al documento de evaluación del terremoto del 13 de enero de 2001). México: Comisión Económica para América Latina y el Caribe.

4. Durkin, M., Khan, N., Davidson, L., Zaman, S. \& Stein, Z. (1993). The effects of a natural disaster on child behaviour: Evidence for posttraumatic stress. Am J Public Health, 83, 1549-1553).

5. Inter Agency Standing Committee. (2007). Guía del IASC sobre salud mental y apoyo psicosocial en emergencias humanitarias y catástrofes. Ginebra: Inter Agency Standing Committee

6. Noji, E. (2000). Impacto de los desastres en la salud pública. Colombia: Organización Panamericana de la Salud.

7. Organización Panamericana de la Salud. (2002). Protección de la salud mental en situaciones de desastres yemergencias. Washington D.C.: OrganizaciónPanamericana de la Salud

8. Organización Panamericana de la Salud. (2002). Terremotos en El Salvador 2001. Crónicas de desastres. Washington D.C.: Organización Panamericana de la Salud.

9. Organización Panamericana de la Salud. (2005). Guía de preparativos de salud frente a erupciones volcánicas. Ecuador: Organización Panamericana de la Salud.

10. Organización Panamericana de la Salud. (2006). Guía práctica de salud mental en desastres. Washington D.C.: Organización Panamericana de la Salud.

11. Organización Panamericana de la Salud. (2010). Apoyo psicosocial en emergencias y desastres. Panamá: Organización Panamericana de la Salud. 
12. Organización Panamericana de la Salud. (2010). Protección de la salud mental luego del terremoto y tsunami del 27 de febrero de 2010 en Chile: crónica de una experiencia. Santiago de Chile: Organización Panamericana de la Salud.

13. Organización Panamericana de la Salud. (2010). Terremoto de Pisco - Perú. A dos años del sismo, crónica y lecciones aprendidas en el sector salud. Perú: Organización Panamericana de la Salud.

14. Pan American Health Organization. (2011). Earthquake in Haiti - One year later. PAHO/WHO report on the health situation. Washington: Pan American Health Organization.
15. Reacher, M., McKenzie, K., Lane, C., Nichols, T. \& Kedge, I. (2004). Health impacts of flooding in Lewes: A comparison of reported gastrointestinal and other illness and mental health in flooded and non-flooded households. Commun Dis Public Health, 7, 39-46.

16. Zeballos, J., Meli, R., Vilchis, A. \& Barrios, L. (1996). The effects of volcanoes on health: preparedness in Mexico. World Health Stat Q, 49 (34), 204-8. 